\title{
Mapping crustal magnetic fields at Mars using electron reflectometry
}

\author{
R. J. Lillis, ${ }^{1}$ D. L. Mitchell, and R. P. Lin $^{1}$ \\ Space Sciences Laboratory, University of California, Berkeley, California, USA
}

J. E. P. Connerney and M. H. Acuña

NASA Goddard Space Flight Center, Greenbelt, Maryland, USA

Received 6 April 2004; revised 11 June 2004; accepted 17 June 2004; published 7 August 2004.

[1] We present a kinetic model for the interaction of electrons precipitating along magnetic field lines into Mars' atmosphere and develop a technique for detecting crustal magnetic fields, which act as magnetic mirrors, reflecting some of the incident electrons back to spacecraft altitudes. We present an initial map covering $20 \%$ of the planet, which shows the topology of magnetic sources along the dichotomy boundary and reveals new magnetic sources in the northern lowlands, implying the existence of preserved Noachian crust beneath at least some regions of the apparently young plains. INDEX TERMS: 5440 Planetology: Solid Surface Planets: Magnetic fields and magnetism; 5443 Planetology: Solid Surface Planets: Magnetospheres (2756); 5421 Planetology: Solid Surface Planets: Interactions with particles and fields; 5409 Planetology: Solid Surface Planets: Atmospheres-structure and dynamics; 5109 Physical Properties of Rocks: Magnetic and electrical properties. Citation: Lillis, R. J., D. L. Mitchell, R. P. Lin, J. E. P. Connerney, and M. H. Acuña (2004), Mapping crustal magnetic fields at Mars using electron reflectometry, Geophys. Res. Lett., 31, L15702, doi:10.1029/2004GL020189.

\section{Introduction}

[2] One of the great surprises of the Mars Global Surveyor mission was the discovery of intensely magnetized crust [Acuña et al., 1999, 2001]. These magnetic sources are at least ten times stronger than their terrestrial counterparts, probably requiring large volumes of magnetized material, very strong remanence, or both [Connerney et al., 1999]. With few exceptions, the crustal fields are associated with the oldest terrain on Mars, and there is evidence for impact demagnetization of the Hellas, Argyre, and Isidis basins, indicating that Mars' crustal magnetic fields are among the oldest preserved geologic features on the planet. Much of the northern lowlands appear to be non-magnetic, except for the relatively weak north polar anomalies and a few sources adjacent to the dichotomy boundary [Acuña et al., 1999], which appear to be associated with strongly magnetized crust south of the boundary.

[3] The MGS Magnetometer has measured crustal magnetic fields at altitudes from 100 to $>1000 \mathrm{~km}$. Because of the long duration in the mapping orbit, the crustal field map at $400 \mathrm{~km}$ altitude is fully sampled on both the day and night hemispheres. However, the sampling is sparse at lower

\footnotetext{
${ }^{1}$ Also at Physics Department, University of California, Berkeley, California, USA.

Copyright 2004 by the American Geophysical Union. 0094-8276/04/2004GL020189\$05.00
}

altitudes, and nearly all of those data were obtained on the sunlit hemisphere, where the solar wind distorts crustal fields, and obscures those weaker than $\sim 10 \mathrm{nT}$ [Brain et al., 2003]. Thus, weaker crustal fields may have so far escaped detection.

[4] The MGS Electron Reflectometer (ER) was designed to remotely probe crustal magnetic fields using the magnetic mirror effect, that is, the reflection of charged particles from regions of increased magnetic field strength [see Acuña et al., 1992]. Comparison of the incident and reflected electron fluxes provides information about the field strength at altitudes where electrons are absorbed by the atmosphere (170-240 km for 190-eV electrons). Electron reflectometry thus provides an independent measure of the field magnitude at altitudes well below that of the spacecraft and closer to the source, where crustal fields are substantially stronger. This technique has been successfully used to map crustal magnetic fields on the Moon [Lin et al., 1998; Halekas et al., 2001; Mitchell et al., 2004]; Here we introduce an adaptation of the technique for a planet with a significant atmosphere. We present an initial map showing the strength and topology of crustal magnetic fields over a region spanning $>20 \%$ of the Martian surface, including both highland and lowland terrains.

\section{Electron Reflectometry}

\subsection{Data Description}

[5] The ER is a hemispherical imaging electrostatic analyzer that samples electron fluxes in sixteen $22.5^{\circ} \times$ $14^{\circ}$ sectors, spanning a $360^{\circ} \times 14^{\circ}$ field of view [Mitchell et al., 2001]. Electron fluxes in each sector are measured in 19 energy channels ranging from $10 \mathrm{eV}$ to $20 \mathrm{keV}$. For this study, we use the 191-eV channel (spanning 143-239 eV), where the count rate is high and the effects of spacecraft charging are negligible.

[6] With knowledge of the magnetic field vector measured onboard, the FOV is mapped into pitch angle $(\alpha)$, which is the angle between an electron's velocity and the magnetic field. During a 2- to 8-sec integration, the ER measures between $8 \%$ and $100 \%$ of the $0^{\circ}-180^{\circ}$ pitch angle spectrum, depending on the orientation of the magnetic field with respect to the FOV plane. The large volume of mapping orbit data contains $>10^{6}$ spectra with sufficient pitch angle coverage for analysis.

\subsection{Kinetic Model}

[7] In a uniform field, electrons move along helical paths of constant radius and pitch angle. However, if the field strength $(B)$ increases towards the planet and the fractional 


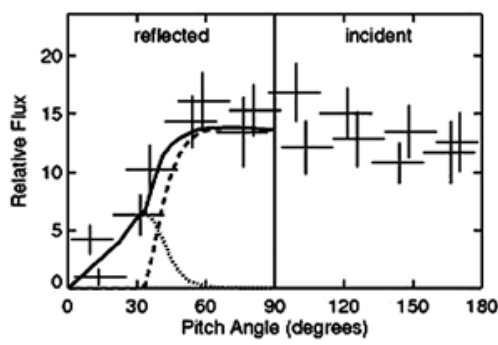

Figure 1. Example 190-eV electron flux in 16 pitch angle bins. Horizontal bars give the bin widths, while vertical error bars include both statistical and calibration uncertainties. A best-fit Monte Carlo kinetic model (solid black curve) is the sum of magnetically reflected (dashed) and backscattered (dotted) electrons.

change in the field is small over the distance traveled by an electron in one gyration, then the adiabatic approximation holds $\left(\sin ^{2} \alpha / B=\right.$ constant $)$, and electrons will be reflected back along the lines of force if $\alpha$ reaches $90^{\circ}$. If there is no interaction with the atmosphere, then the reflected electrons return to the spacecraft after a round-trip time of $\sim 0.1 \mathrm{sec}$ with the same energy and a pitch angle of $180^{\circ}-\alpha$, according to the adiabatic condition.

[8] The magnetic field strength from crustal sources increases towards the planet. Thus, downward traveling electrons with pitch angles near $90^{\circ}$ at the spacecraft reflect at high altitudes, while those with pitch angles closer to $0^{\circ}$ $\left(\right.$ or $180^{\circ}$ ) at the spacecraft reflect at lower altitudes. Since the atmospheric density increases exponentially towards the surface, the probability that an electron will impact a neutral species increases rapidly as the reflection point gets lower. Most scattered electrons do not return to the spacecraft, so the flux of upward traveling electrons exhibits an attenuation, or loss cone, that depends sensitively on pitch angle. Impacting electrons are scattered in both angle and energy, so that the adiabatic condition no longer applies. Some of these scattered electrons return to the spacecraft, partially filling the loss cone and mixing with magnetically reflected electrons. Since only the adiabatic population retains information about the magnetic field at lower altitudes, it is first necessary to separate the adiabatic and backscattered populations. This is possible because the two populations have distinct, albeit overlapping, pitch angle distributions.

\subsection{Backscattered Electron Population}

[9] We have developed a Monte Carlo model to simulate the backscattered electron opulation under various conditions. For each of 40 initial pitch angles, 1000 particles with a typical energy distribution from $80 \mathrm{eV}$ to $20 \mathrm{keV}$ [Mitchell et al., 2001] are sent down a magnetic field line into an MTGCM atmosphere [Bougher et al., 1999, 2000]. Over 500 types of elastic and inelastic collisions with $\mathrm{CO}_{2}, \mathrm{O}, \mathrm{CO}$, $\mathrm{O}_{2}$, and $\mathrm{N}_{2}$ are included, using cross sections compiled by Sung and Fox [2000], Shyn and Sharp [1979] and Burnett and Rountree [1979]. For each electron, the probabilities for all of these collisions are calculated as a function of distance along the electron's spiral path, and random numbers are used to determine a) where (if at all) a collision occurs, b) if a collision occurs, the amount of energy lost by the electron and c) the scattering angle according to phase functions derived from theory and laboratory experiments [Porter and
Jump, 1978; Porter et al., 1987]. A new helical trajectory is calculated, and the Monte Carlo procedure is repeated until the particle is either lost or returns to the spacecraft. For our purposes, an electron is "lost" when its energy falls below the acceptance range of the $191-\mathrm{eV}$ energy channel. This procedure accounts for cascading from higher energy channels into the $191-\mathrm{eV}$ channel.

[10] Backscattered electrons partially fill the loss cone, shifting the pitch angle at which it begins to form by several degrees (Figure 1). We performed Monte Carlo simulations for a variety of magnetic field gradients and dip angles (the angle the field makes with the local horizontal) to observe how these parameters affected the backscatter distribution and the loss cone position. For convenience, we define $\alpha_{C}$ to be the critical pitch angle at which the upward flux falls to $60 \%$ of the downward flux. We found that, to a good approximation, $\alpha_{C}$ uniquely determines the pitch angle distribution of backscattered electrons. Thus, these simulations comprise a lookup table for estimating the backscatter distribution for any measured value of $\alpha_{C}$. After subtracting backscatter from the measured pitch angle distributions, we can then use a much simpler adiabatic code, which is $10^{6}$ times faster, for least-squares analysis of the entire data set.

\subsection{Adiabatic Electron Population}

[11] Once the backscatter population is removed, the problem is reduced to one of calculating the survival probability for electrons traveling from the spacecraft to the reflection point and back. (Once an electron suffers any type of collision it is lost.) To first order, the collision probability, $P$, depends on the collision cross-sections, $\sigma_{i}$, and the path-integrated densities, $\left\langle n_{i}\right\rangle$, of each of the atmospheric constituents along a helical trajectory of length $L$ from the spacecraft to the reflection point and back:

$$
P=1-\exp \left(-L \sum_{i}\left\langle n_{i}\right\rangle \sigma_{i}\right) ; \quad\left\langle n_{i}\right\rangle=\frac{2}{L} \int_{0}^{x_{r}} n_{i}(x) \sec [\alpha(x)] d x
$$

where $\alpha$ is the pitch angle at position $x$ along the field line, $x_{r}$ is the value of $x$ at the reflection point, and the factor of two accounts for the round trip through the same column of atmosphere. With the adiabatic approximation, $P$ becomes:

$$
P=1-\exp \left[-2 \int_{0}^{x_{r}} \frac{\sum_{i} \sigma_{i} n_{i}(x) d x}{\sqrt{1-\frac{B(x)}{B_{s c}}} \sin ^{2} \alpha_{s c}}\right] .
$$

where $B(x)$ is the field magnitude at $x$ and $B_{s c}, \alpha_{s c}$ are the measured field and the pitch angle at the spacecraft. In order to calculate $P$, we must know the collision cross sections, as well as the neutral density and magnetic field strength as a function of $x$. Electron impact cross-sections are known to within $20 \%$ [Sung and Fox, 2000], so these are held fixed throughout the analysis. Thermospheric global circulation models, such as the MTGCM [Bougher et al., 1999, 2000], make predictions for the lower exospheric density, which vary with season and solar cycle and are thought to be correct to within a factor of $2-3$. For this study, which includes data from April 1999 through October 2003, we 

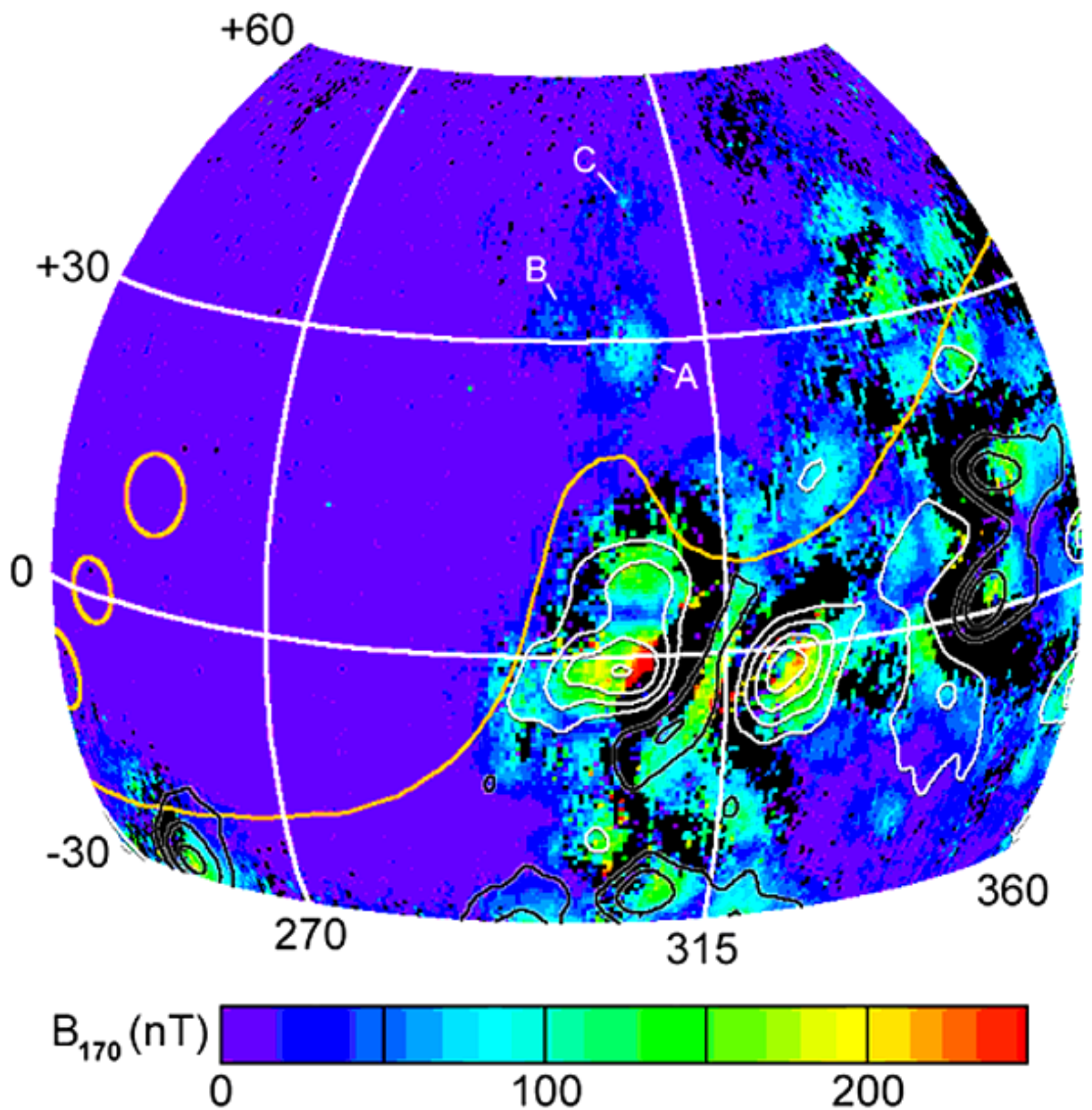

Figure 2. The crustal magnetic field strength at $170 \mathrm{~km}$ altitude, as inferred from electron reflectometry, is averaged in $0.5^{\circ} \times 0.5^{\circ}$ bins (color scale). All data were obtained at 2 a.m. local time. Double-sided loss cones were observed in black regions within $45^{\circ}$ of the equator, indicating that the spacecraft was on closed crustal magnetic field lines (miniature magnetospheres). At higher latitudes, black patches may also indicate sparse sampling. Contours show the radial component of the magnetic field measured by the MAG at $400 \mathrm{~km}$ [Connerney et al., 2001] (white: negative, black: positive). The locations of the Tharsis volcanoes and the dichotomy boundary are shown in orange.

adopt an MTGCM atmosphere corresponding to the middle of the solar cycle with Mars at equinox and assume diffusive equilibrium to extrapolate densities from the top of the model to $400 \mathrm{~km}$. The crustal magnetic field is known with varying degrees of accuracy, depending on its strength. Where the field is strongest, spherical harmonic expansions accurately represent $B(x)$ from $170 \mathrm{~km}$ to $400 \mathrm{~km}$ altitude [Arkani-Hamed, 2001; Cain et al., 2003], and we can use the electron data to further constrain the atmospheric density [Lillis et al., 2003]. For this study, however, we are concerned with regions where the crustal field is known to be weak ( $<10 \mathrm{nT}$ at $400 \mathrm{~km})$ but is otherwise unconstrained.

\section{Mapping Weak Crustal Fields}

[12] On the night hemisphere over regions where the crustal magnetic field is weak, the field at the spacecraft is dominated by the draped interplanetary magnetic field (IMF), which has a small gradient between 400 and $170 \mathrm{~km}$ [Ma et al., 2002], resulting in a loss cone with 
$\alpha_{C} \approx 90^{\circ}$. Crustal magnetic fields are revealed whenever they connect with the IMF and cause $\alpha_{C}$ to depart from $90^{\circ}$. Our technique does not apply to closed crustal magnetic field loops, where trapped electrons can make multiple reflections through the atmosphere, creating double-sided loss cones (at $\alpha_{C}$ and $180^{\circ}-\alpha_{C}$ ).

[13] We calculate representative values for the strengths of these weak crustal fields by parameterizing the altitude variation of the magnetic field. For simplicity, we represent the total field, $B(x)$, by a constant term (the solar windinduced field, $B_{s w}$ ) plus a power law with exponent $a$ (the crustal field), and we assume that the field is a straight-line extension of the field vector measured at the spacecraft Our parameterization then becomes:

$$
B(x)=B_{s w}+\left(B_{s c}-B_{s w}\right)\left(\frac{x_{s}}{x}\right)^{a}
$$

where $x_{s}$ is the distance along the field line from the spacecraft to the crustal source, which we assume to be $15 \mathrm{~km}$ below the surface, or half the estimated depth of the historical Curie isotherm [Arkani-Hamed, 2003].

[14] The two unknown parameters, $B_{s w}$ and $a$, are correlated, so we assume a nominal exponent of 2.5 , which is a compromise between an infinite line of dipoles $(a=2)$ and an isolated dipole $(a=3)$. The effective power law exponent over the strong magnetic sources in Terra Sirenum, for example, is $\sim 2.2$ [Brain et al., 2003]. We then fit the adiabatic model (equation (2)) to the measured pitch angle distribution to find the value of $B_{s w}$ that minimizes $\chi^{2}$. Finally, we use equation (3) to estimate the crustal field strength at altitudes below the spacecraft.

[15] Figure 2 shows the field strength due to crustal sources at an altitude of $170 \mathrm{~km}\left(B_{170}\right)$, which is near the bottom of the altitude range probed by $190-\mathrm{eV}$ electrons. Variation over reasonable ranges of our assumed parameters a (2 to 3 ) and $n_{i}$ (MTGCM solar min-aphelion to solar maxperihelion), uncertainties in fitting the distributions and differing densities of data points in each pixel yield a typical error of $\sim 35 \%$ in $B_{170}$. Interference from changing IMF polarity and weak gradients in the solar wind-induced field obscure crustal fields smaller than $\sim 5 \mathrm{nT}$. Over the strongest crustal sources, where a reliable comparison can be made between our map and low-altitude dayside MAG data, we find agreement to within $35 \%$ at $170 \mathrm{~km}$.

[16] The ER map shows two new sources in Tempe Terra (B and $\mathrm{C}$ in Figure 2) and more completely maps a third source (A) that was first detected during aerobraking. The ER map also reveals crustal field topology, including regions of closed crustal magnetic field loops that form curved patterns extending over hundreds of kilometers. These patterns smoothly cross the dichotomy boundary east of $\sim 300^{\circ}$ longitude. The presence of magnetic sources well north of the dichotomy boundary suggests that ancient crust is preserved in a significant fraction of the northern lowlands. This is compatible with the presence of buried craters inferred from MOLA topographic data [Frey et al., 2002], which provide strong evidence that the northern lowland plains overlie a much older surface. In contrast, there is no evidence for magnetic sources over most of the Tharsis rise down to our detection threshold (with the notable exception of its southern boundary). We are currently extending the coverage of Figure 2 globally.
[17] Acknowledgments. We thank M. Liemohn for many helpful discussions. This work was supported by NASA Grant JPL1233751.

\section{References}

Acuña, M. H., et al. (1992), The Mars Observer magnetic fields investigation, J. Geophys. Res., 97, 7799.

Acuña, M. H., et al. (1999), Global distribution of crustal magnetism discovered by the Mars Global Surveyor MAG/ER experiment, Science, 284,790 .

Acuña, M. H., et al. (2001), Magnetic field of Mars: Summary of results from the aerobraking and mapping orbits, J. Geophys. Res., 106, 23,403.

Arkani-Hamed, J. (2001), A 50-degree spherical harmonic model of the magnetic field of Mars, J. Geophys. Res., 196, 23,197.

Arkani-Hamed, J. (2003), Thermoremanent magnetization of the Martian lithosphere, J. Geophys. Res., 108(E10), 5114, doi:10.1029/2003JE002049.

Bougher, S. W., S. Engle, R. G. Roble, and B. Foster (1999), Comparative terrestrial planet thermospheres: 2 . Solar cycle variation of global structure and winds at equinox, J. Geophys. Res., 104, 16,591.

Bougher, S. W., S. Engle, R. G. Roble, and B. Foster (2000), Comparative terrestrial planet thermospheres: 3 . Solar cycle variation of global structure and winds at solstices, J. Geophys. Res., 105, 3091.

Brain, D. A., F. Bagenal, M. H. Acuña, and J. E. P. Connerney (2003), Martian magnetic morphology: Contributions from the solar wind and crust, J. Geophys. Res., 108(A12), 1424, doi:10.1029/2002JA009482.

Burnett, T., and S. P. Rountree (1979), Differential and total cross sections for electron-impact ionization of atomic oxygen, Phys. Rev. A, 20, 1468.

Cain, J. C., B. B. Ferguson, and D. Mozzoni (2003), An n $=90$ internal potential function of the Martian crustal magnetic field, J. Geophys. Res., 108(E2), 5008, doi:10.1029/2000JE001487.

Connerney, J. E. P., M. H. Acuña, P. J. Wasilewski, N. F. Ness, H. Réme, C. Mazelle, D. Vignes, R. P. Lin, D. L. Mitchell, and P. A. Cloutier (1999), Magnetic lineations in the ancient crust of Mars, Science, 284, 794.

Connerney, J. E. P., M. H. Acuña, P. Wasilewski, G. Kletetschka, N. F. Ness, H. Reme, R. P. Lin, and D. Mitchell (2001), The global magnetic field of Mars and implications for crustal evolution, Geophys. Res. Lett., 28,4015 .

Frey, H. V., J. H. Roark, K. M. Shockey, E. L. Frey, and S. E. H. Sakimoto (2002), Ancient lowlands on Mars, Geophys. Res. Lett., 29(10), 1384, doi:10.1029/2001GL013832.

Halekas, J., D. L. Mitchell, R. P. Lin, S. Frey, L. L. Hood, M. H. Acuña, and A. B. Binder (2001), Mapping of crustal magnetic anomalies on the lunar near side by the Lunar Prospector magnetometer/electron reflectometer, J. Geophys. Res., 106, 27,841.

Lillis, R. J., D. L. Mitchell, R. P. Lin, and M. H. Acuña (2003), Measuring Mars' atmospheric neutral density from 160 to $320 \mathrm{~km}$ altitude with the MGS electron reflectometer, Eos Trans. AGU, 84(46), Fall Meet. Suppl., P52B-05.

Lin, R. P., D. L. Mitchell, D. W. Curtis, K. A. Anderson, C. W. Carlson, J. McFadden, M. H. Acuña, L. L. Hood, and A. Binder (1998), Lunar surface magnetic fields and their interaction with the solar wind: Results from Lunar Prospector, Science, 281, 1480.

Ma, Y., A. F. Nagy, K. C. Hansen, D. L. DeZeeuw, T. I. Gombosi, and K. G. Powell (2002), Three-dimensional multispecies MHD studies of the solar wind interaction with Mars in the presence of crustal fields, J. Geophys. Res., 107(A10), 1282, doi:10.1029/2002JA009293.

Mitchell, D. L., R. P. Lin, C. Mazelle, H. Rème, P. A. Cloutier, J. E. P. Connerney, M. H. Acuña, and N. F. Ness (2001), Probing Mars' crustal magnetic field and ionosphere with the MGS Electron Reflectometer, J. Geophys. Res., 106, 23,419.

Mitchell, D. L., J. S. Halekas, R. P. Lin, S. Frey, L. L. Hood, M. H. Acuña, and A. Binder (2004), Global mapping of lunar crustal magnetic fields by Lunar Prospector, Icarus, in press.

Porter, H. S., and F. W. Jump (1978), Analytic total angular elastic electron impact cross sections for planetary atmospheres, NASA Tech. Memo., CSC/TM-78/6017, 23 pp.

Porter, H. S., F. Varosi, and H. G. Mayr (1987), Iterative solution of the multistream electron transport equation 1: Comparison with laboratory beam injection experiments, J. Geophys. Res., 92, 5933.

Shyn, T. W., and W. E. Sharp (1979), Doubly differential cross section of secondary electrons ejected from gases by electron impact: $50-400 \mathrm{eV}$ on $\mathrm{CO}_{2}$, Phys. Rev. A, 20, 2332.

Sung, K., and J. L. Fox (2000), Electron impact cross sections for use in modeling the ionospheres/thermospheres of the earth and planets, EOS Trans. $A G U, 81(48)$, Fall Meet. Suppl., SA52A-11.

M. H. Acuña and J. E. P. Connerney, NASA-GSFC, Greenbelt, MD 20771, USA.

R. J. Lillis, R. P. Lin, and D. L. Mitchell, Space Sciences Laboratory, University of California, Berkeley, CA 94720, USA. (rlillis@ssl.berkeley. edu) 